\title{
Where are correlations hidden in the distribution of the largest fragment?
}

\author{
Robert Botet* \\ Laboratoire de Physique des Solides Bât.510 - CNRS UMR8502 / Université Paris-Sud, Centre \\ d'Orsay, F-91405 Orsay, France \\ E-mail: robert.botet@lps.u-psud.fr
}

\begin{abstract}
For systems broken in pieces, an immediate observable is the size of the largest fragment. Despite the apparent simplicity of this variable, one can extract definite information about the correlations present in the system, and even the mechanisms which led to this special fragment. The underlying idea is that the largest fragment is indeed very special, because, during the history of its formation, this fragment avoided (to some extent) processes of fragmentation and most of the relevant correlations. However, to get the useful information, average values are not enough, and one has to investigate in details the complete fluctuations of the largest-fragment size, under the form of its probability-distribution function. Focusing here on principles and not on particular applications, we take two simple models of aggregation exhibiting critical behavior (at-equilibrium percolation and dynamical Smoluchowski equations), we show and discuss in the present work the way the information is hidden in the largest-fragment distribution. Moreover, the distribution of the second largest fragment gives precise insights about the mechanisms which generated the system. This approach provides a novel kind of data analysis, which can be helpful when one has only access to the final state of a system of fragments, as it may happen in nuclear collisions or in explosion of hot atomic clusters.
\end{abstract}

The Seventh Workshop on Particle Correlations and Femtoscopy

September 20 - 242011

University of Tokyo, Japan

\footnotetext{
${ }^{*}$ Speaker.
} 


\section{Introduction}

In the $N$-body system, correlations reflect the interactions between particles. However, it may happen that these quantities are not directly available in the experiments. In such a case, one has to disentangle indirect information to obtain results about the underlying correlations. A way to do that - though barely used - is the detailed investigation of the probability distribution function of a relevant observable, such as the order parameter [1,2]. As a matter of fact, the logarithm of the order-parameter distribution is nothing but the free energy of the system, which is known to include all the relevant information about the correlations [3].

In the simplest example of the additive observable (e.g. the magnetization), the Central Limit Theorem assures that the corresponding probability distribution must be a gaussian function provided correlations be unessential [4]. Therefore, any deviation to the gaussian shape results, in this case, from correlations. We describe below how to use a similar idea in the less-known example of the extremal variable, which is the usual case when system is made of fragments.

\section{The extremal variable}

Percolation is the paradigm of systems broken in pieces [5]. A particular phase transition occurs between two phases: the ordered phase and the disordered phase. These two phases are respectively characterized by the occurrence or not of a giant macroscopic piece, coexisting with an ensemble of microscopic fragments. The size of the biggest fragment appears to be the natural order parameter in this case [6]. If $s_{i}$ denotes the size of the fragment number $i$, and there are $m$ fragments in the system, the order parameter, $s_{\max }$, is then defined as:

$$
s_{\max }=\max _{i=1, \cdots, m} s_{i} .
$$

The number $m$ is also called the multiplicity of the system.

When there is no correlations in the system, the fragments are independent one each other. It means that the fragment sizes, $s_{i}$, are independent random variables with a common distribution function, $f(s)$, which reflects the manner the fragments have been built. Then, the probability distribution of the extremal variable $s_{\max }$ is given by a Limit Theorem, essentially due to Gnedenko in its modern form [7]. According to special features of the distribution function $f(s)$, the random variable $s_{\max }$ for a large number of fragments, is distributed following $\phi\left(s_{\max }\right)$, which must be either the Gumbel distribution, or the Fréchet distribution or the Weibull distribution [8]. No other distribution can appear for the extremal variable in the uncorrelated system.

For brevity, we will consider below the Gumbel distribution, as it is the most general case, though the two other cases should be worth detailed investigation.

\subsection{The Limit Theorem for extremal variables (Gumbel case)}

Suppose that the multiplicity, $m$, is a large constant number, and the following condition on the fragment-size distribution, $f(s)$, is asymptotically fulfilled [9]:

$$
f(s)+\frac{d f(s)}{d s} \int_{s}^{\infty} f\left(s^{\prime}\right) d s^{\prime} \rightarrow 0 \quad \text { when } s \rightarrow \infty,
$$


then, there exist two quantities $a_{m}$ and $b_{m}>0$, depending on the multiplicity and on some features of the function $f(s)$ (we shall be more precise in the next section), for which the probability distribution $\phi_{1}$ of the extremal variable $s_{\max }$ writes:

$$
\begin{aligned}
\phi_{1}\left(s_{\max }\right) & =\frac{1}{b_{m}} e^{-z_{1}-e^{-z_{1}}}, \\
z_{1} & =\frac{s_{\max }-a_{m}}{b_{m}}
\end{aligned}
$$

and moreover, the coefficients $a_{m}$ and $b_{m}$ are such that [10]:

$$
\int_{a_{m}}^{\infty} f\left(s^{\prime}\right) d s^{\prime}=\frac{1}{m} ; b_{m}=\frac{1}{m f\left(a_{m}\right)} .
$$

The demonstration of this theorem is straightforward, starting from the relation: $\operatorname{prob}\left[s_{\max } \leq\right.$ $\left.s^{\prime}\right]=\left(\operatorname{prob}\left[s \leq s^{\prime}\right]\right)^{m}$, which expresses that the condition for $s_{\max }$ to be smaller than a value $s^{\prime}$ is that all the fragment sizes are smaller than $s^{\prime}$.

The normalized double-exponential function (2.3) is the Gumbel distribution [11]. This function behaves as a simple exponential function, $\sim \exp \left(-s_{\max } / b_{m}\right)$, for the large positive values of the argument, but decreases sharply as a double-exponential function, $\sim \exp \left(-\exp \left(-\left(s_{\max }-\right.\right.\right.$ $\left.\left.a_{m}\right) / b_{m}\right)$ ) on the other side, when $s_{\max }-a_{m} \ll 0$.

\subsection{Examples of distributions belonging to the domain of attraction of the Gumbel distribution}

Here is listed a couple of distributions $f(s)$ which meet the condition (2.2), and the corresponding attraction coefficients $a_{m}$ and $b_{m}$.

- the exponential distribution:

$$
\begin{aligned}
f(s) & =\frac{1}{s_{0}} e^{-s / s_{0}} \\
a_{m} & =s_{0} \ln m ; b_{m}=s_{0}
\end{aligned}
$$

- the gaussian distribution:

$$
\begin{aligned}
f(s) & =\frac{1}{\sqrt{2 \pi s_{0}^{2}}} e^{-(s-\mu)^{2} / 2 s_{0}^{2}} \\
a_{m} & =s_{0} \sqrt{2 \ln m} ; b_{m}=s_{0} / \sqrt{2 \ln m}
\end{aligned}
$$

According to the condition (2.2), only the large-s tail of $f(s)$ matters in the definition of the domain of attraction, such that it is sufficient that the behaviors (2.6) or (2.8) are asymptotically true for the conclusions of the Limit Theorem be valid. This is the case for most of the cut-offs [12], hence the ubiquity of the Gumbel distribution in the physics of extremal variables.

One should note on these simple examples, the particular meaning of the ratio $s / s_{0}$. Actually, if one believes the exponential decrease as the most plausible cut-off, it appears that $b_{m}$ is a measure of the correlation size [13] ( $s_{0}$ in Equ.(2.6)). 


\subsection{Role of the multiplicity fluctuations}

In the real situations, there is no reason why the multiplicity should be a constant number. However, a simple argument can be given to prove the stability of the distribution when gaussian fluctuations of $m$ are considered. Let us consider the binomial distribution of the multiplicity, that is:

$$
P[m]=\left(\begin{array}{c}
M \\
m
\end{array}\right) p^{m}(1-p)^{M-m}
$$

with the constant probability, $p$, and the maximum multiplicity, $M$. The average value of the multiplicity is $\langle m\rangle=p M$. Then, the average of the cumulative probability distribution function $\operatorname{prob}\left[s_{\max } \leq s^{\prime}\right]$ over random realizations of $m$, is such that:

$$
\begin{aligned}
\operatorname{prob}\left[s_{\max } \leq s^{\prime}\right] & =\sum_{m=0}^{M} P[m]\left(\operatorname{prob}\left[s \leq s^{\prime}\right]\right)^{m} \\
& \simeq\left(\operatorname{prob}\left[s \leq s^{\prime}\right]\right)^{p M}
\end{aligned}
$$

leading again to the Gumbel distribution following the same line as for the constant- $m$ case. Consequently, the Limit Theorem for the extremal variable, holds identically true when the multiplicity is gaussian-fluctuating, with the replacement: $m \rightarrow\langle m\rangle$.

\section{The percolation model}

Let us come back to the percolation model. This is a geometrical model on a lattice, in which the system is divided in clusters intra-connected with bonds, each bond appearing with a constant probability, say $p$ [5]. There is no time in the model, that is the system is expected to be at the thermodynamic equilibrium [6]. When $p$ is smaller than a threshold $p_{c}$, all the clusters remain microscopic in size (in the sense: $\left\langle s_{\max }\right\rangle / N \rightarrow 0$, when the size $N$ of the lattice tends to $\infty$ ), while a macroscopic cluster $\left(\left\langle s_{\max }\right\rangle\right.$ is a finite fraction of the total mass $N$ ) appears when $p>p_{c}$ and spans the lattice. The threshold $p=p_{c}$ is the critical point for the infinite system. In this model, the natural order parameter is the probability for a given site to belong to the spanning cluster. Its alternative form is: $s_{\max } / N$ - ratio between the size of the largest cluster and the size of the lattice -, since the percolating cluster is almost surely the largest cluster in the system. We discuss hereafter a few results known about the distribution of the order parameter in the percolation model.

\section{1 percolation - the disordered phase $p<p_{c}$}

When $p<p_{c}$, the distribution of the largest-cluster size was proved to follow a Gumbel distribution $[14,15]$. The proof is either numerical or based on the renormalization group theory. This result is consistent with the general theory explained above, which states that the distribution of the extremal variable is the Gumbel distribution as long as the correlations are irrelevant.

It is the place here to give details about what 'irrelevant correlations' means exactly. Indeed, correlations are present in the system for any finite value of $p$, and a correlation length, $\xi(p)$ is associated. Below the critical threshold, $\xi(p)<\infty$. As the critical state corresponds to appearance of a cluster spanning the maximum system length $L$ (the lattice diameter), correlations are irrelevant as long as the condition: $\xi(p)<L$ is realized. On the other hand, this condition defines a particular 
value, $p_{\star}$, through the implicit equation $\xi\left(p_{\star}\right)=L$. In other words, $p_{\star}$ corresponds to the value of $p$ for which the correlation length is comparable to the system diameter. Actually, the interval $\left[p_{\star}, p_{c}\right]$ forms the lower part of a domain in $p$, called the pseudo-critical domain [16]. In the pseudo-critical domain, the finite system resembles the critical infinite system, in the sense that the correlation length exceeds the system size.

\section{2 percolation - the critical phase $p=p_{c}$}

The system undergoes a continuous phase transition for the threshold value $p=p_{c}$. One knows that the universality class of the system can be characterized by the values of two critical exponents [17]. For the percolation model, these exponents are either known numerically (finite space dimension $>2$ ) or exactly, in the mean-field case and for the 2-dimensional space.

Little is known about the distribution of the order parameter in the critical percolation network, except a remarkable exact result in the mean-field case [18]. Indeed, for the percolation on the Bethe lattice at $p=p_{c}$, with $p_{c}$ the critical value of the probability $p$ for the infinite lattice, it was shown that the distribution of the order parameter, $s_{\max } / N$, is the Kolmogorov-Smirnov distribution [19], conveniently written in terms of its moment-generating function (Laplace transform):

$$
\begin{aligned}
\phi_{1}\left(s_{\max }\right) & =\frac{1}{c_{N}} f_{1}\left(\frac{s_{\max }}{c_{N}}\right) \\
c_{N} & \sim \ln ^{2} N \\
\int_{0}^{\infty} e^{-t z} f_{1}(z) d z & =\frac{\sqrt{6 t}}{\sinh \sqrt{6 t}}
\end{aligned}
$$

The function $\phi_{1}$ behaves as a simple exponential function, $\sim \exp \left(-s_{\max } / c_{N}\right)$, for the large positive values of the argument, and decreases sharply as the function: $\sim \exp \left(-c_{N} / s_{\max }\right)$, on the other side where $s_{\max } \rightarrow 0$. This special distribution does not appear to be the limit in some way, of the Gumbel distribution [20].

\section{3 percolation - the ordered phase $p>p_{c}$}

The ordered phase is characterized by the giant cluster spanning the entire lattice, and correlations going exponentially fast to their limit value. Similarly to the disordered case, one can define a special value $p^{\star}$, such that: $\xi\left(p^{\star}\right)=L$. This value characterizes the upper limit of the pseudo-critical domain.

However, the situation is quite different when compared with the disordered case. One can catch the difference with the following argument: if we merge two independent finite systems, say: $A$ and $B$, with same size $N$, same value of $p>p_{c}$, and respective order parameters: $s_{\max }(A)$ and $s_{\max }(B)$, the value of the order parameter of the resulting system $A \cup B$ is $s_{\max }(A \cup B)=\left(s_{\max }(A)+\right.$ $\left.s_{\max }(B)\right) / 2 N$, because the spanning cluster of the system $A$ joins the spanning cluster of the system $B$ almost surely. Then, the order parameter is essentially of additive nature, and no more of extremal nature. It results, in this case, in the gaussian distribution of the order parameter.

\section{The critical mean-field aggregation model}

The percolation model is a geometrical at-equilibrium system which exhibits a continuous phase transition of definite universality class. In some cases (for example: the mean-field class) 
there exists a critical dynamical model which belongs to the same universality class. The model is called the Smoluchowski aggregation model [21], since the basic equations were written by Smoluchowski one century ago [22], to investigate the dynamics of coalescence of droplets. The equations write:

$$
\frac{d c_{k}}{d t}=\frac{1}{2} \sum_{i+j=k} K_{0} i j c_{i} c_{j}-\sum_{i=1}^{N} K_{0} i k c_{i} c_{k}
$$

where $c_{k}$ is the concentration of droplets of volume $k$, and $K_{0} i j$ represents the coalescence crosssection of two droplets of respective volumes $i$ and $j . N$ is the total volume of the matter in the system, and the volume of a droplet cannot be smaller than 1 .

Starting from appropriate initial conditions, the $N$ coupled equations (4.1) describe the evolution of the droplets population with the time $t$. One then defines the order parameter as the reduced largest droplet size, $s_{\max } / N$. This quantity exhibits a critical behavior for the particular time $t_{c}=\left(K_{0} \sum_{k} k^{2} c_{k}(0)\right)^{-1}$, named the gel time. More precisely, $\lim _{N \rightarrow \infty} s_{\max } / N$ is finite only when $t>t_{c}$ and a macroscopic phase appears, otherwise this quantity vanishes and the system is made of small clusters.

The critical exponents are known exactly for this model. They coincide with the analogous exponents of the mean-field percolation model, then both models are expected to belong to the same universality class. The probability distribution function of the order parameter in the Smoluchowski model, is not known exactly. However, on the basis of extensive numerical simulations, it was proposed that the $s_{\max }$-distribution function is the Kolmogorov-Smirnov distribution, the same as for the mean-field critical percolation model [18]. Moreover, the distribution is definitely a Gumbel distribution in the disordered phase.

This aggregation model is convenient for numerical simulations. That way, this model is able to provide detailed information about features which are not available otherwise. We exemplify below this ability by investigating the probability distribution of the order parameter in the pseudocritical domain $\left[t_{\star}(N), t^{\star}(N)\right]$, where $t_{\star}(N)<t_{c}$ and $t^{\star}(N)>t_{c}$ are the lower and upper times for which the correlation size is $N$. Precise estimation of the limits of the pseudo-critical domain is not easy to do, as it requires analysis of genuine correlation functions. However, one can propose rough estimations using behaviors of critical quantities. Let us take the example of the standard deviation, $\sigma$, of the largest cluster in the system. $\sigma$ must diverge at the gel time for the infinite system. For the finite systems, $\sigma$ is expected to reach a maximum for a definite value of the time close to $t_{c}$, moreover the maximum must increase with the size of the system. The shape of $\sigma$ versus the time is shown on the Figure 1 for a finite system of size $N=512$. Indeed, the behavior exhibits sharp maximum for the time $t / t_{c} \simeq 1$.3. If we propose that the value of $\sigma$ is 'large' when it is above $10 \%$ of its maximum value, then two times can be defined this way, and their values are respectively $t_{\star}=0.6 t_{c}$ and $t^{\star}=2.3 t_{c}$.

\subsection{Guess for fit of the order-parameter distribution function}

The probability that the system state exhibits the features of the disordered state (i.e. the Gumbel distribution) depends on the distance to the gel time. It must be 1 when $t \leq t_{\star}$, and 0 when 


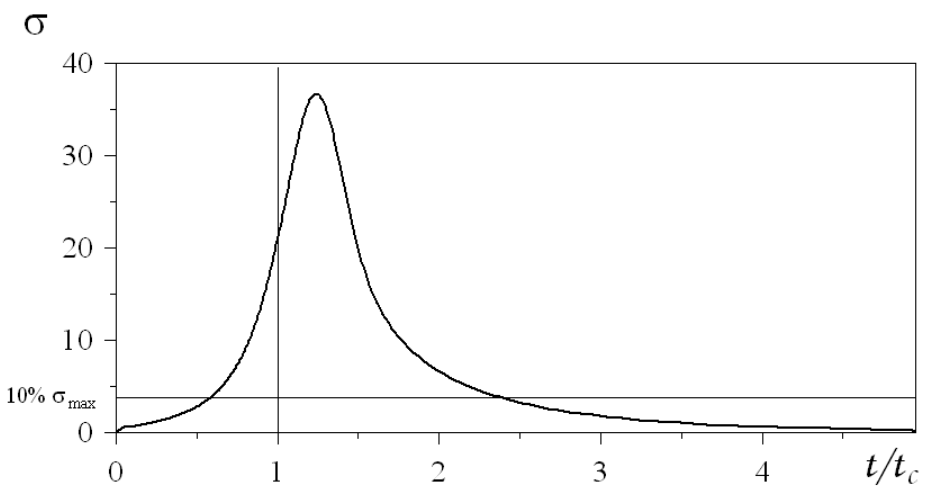

Figure 1: Standard-deviation, $\sigma$, of the largest fragment size versus time $t$, for the finite Smoluchowski aggregation model. The size of the system is $N=512$ and $10^{5}$ independent numerical experiments were used. The maximum value of $\sigma$ is found to be $\sigma_{\max } \simeq 36$ at the time $t=1.3 t_{c}$. The bounds of the pseudocritical domain can be roughly estimated as the times where the values of $\sigma$ are small enough. Here we took the criterion $\sigma=0.1 \sigma_{\max }$. It results in the values $t_{\star}=0.6 t_{c}$ and $t^{\star}=2.3 t_{c}$. The vertical line is the critical time for the infinite system.

$t \geq t^{\star}$. But in the pseudo-critical domain of time, the finite-system state experiences fluctuations large enough to correspond to either the disordered or the ordered phase. This behavior, due to the finite character of the system, must not be confused with a phase coexistence. We are here dealing with state-fluctuations from sample to sample, and not phase-fluctuations inside a given system. This suggests that a natural fit for the effective $s_{\max }$ probability distribution function, is the sum of a Gumbel distribution (disordered state) and of a gaussian distribution (ordered state), with respective weights being function of the distance $t-t_{c}$.

Note that for the finite systems, the special critical distribution at the gel time is not expected to play an important role, as the probability for the system to appear exactly at this point is infinitely small.

\subsection{Results about the order-parameter distribution function in the pseudo-critical domain}

We performed numerical simulations of the Smoluchowski equations (4.1) for finite systems, and analyze the averaged $s_{\max }$-distribution as sums of weighted Gumbel + Gauss distributions.

To realize that task, we estimated the five unknown parameters for every numerical distribution (two parameters for each partial distribution and the relative weight of the Gumbel distribution to the gaussian distribution) using Monte-Carlo $\chi^{2}$-minimization.

- $t_{\star}<t<t_{c}$

In the pre-gelation regime, one should expect the Gumbel distribution to be the dominant part. This is clear on the Figure 2 (a), which corresponds to the time $t=0.4 t_{c}$, laying slightly outside the boundary of the pseudo-critical domain. The probability distribution is almost perfectly fitted with a Gumbel distribution.

On the later time $t=0.8 t_{c}$ (Figure $2(\mathrm{~b})$ ), the role of the gaussian distribution becomes clear as this part grows up progressively in the vicinity of the gel-time. 

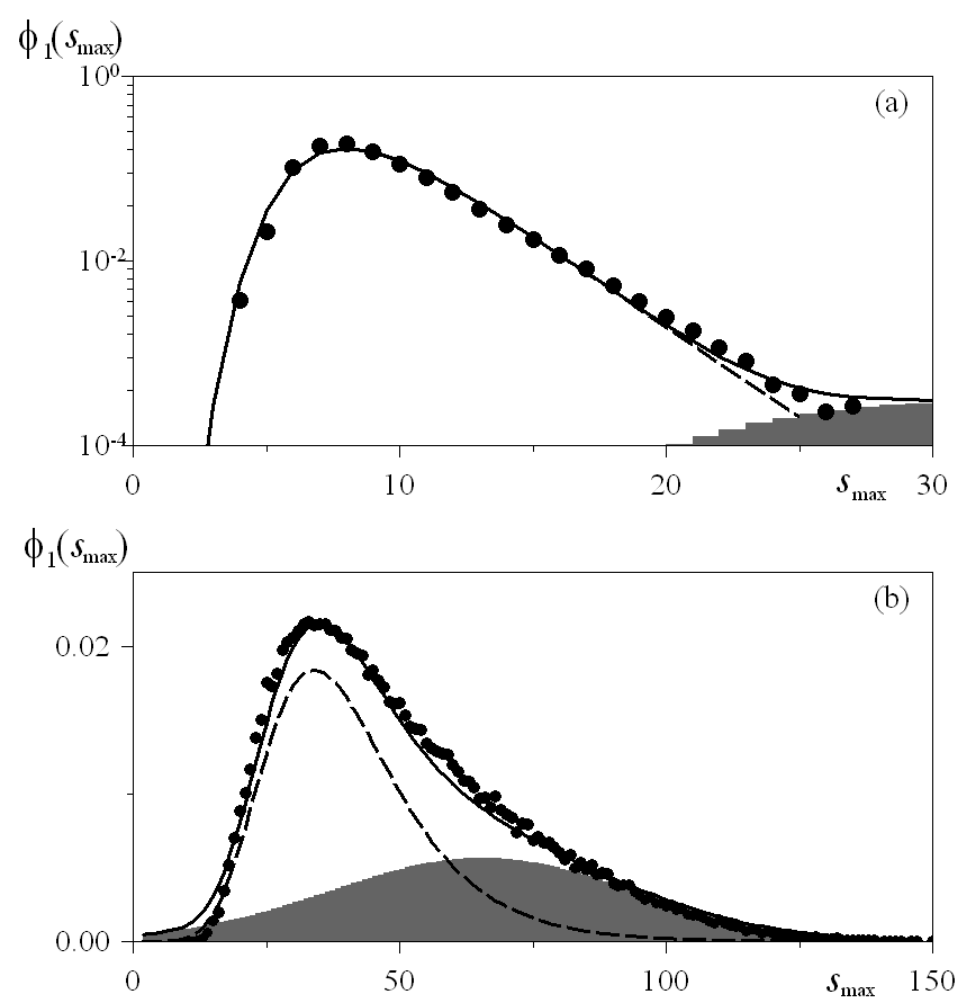

Figure 2: Probability distribution of the order parameter $s_{\max }$ for the Smoluchowski aggregation model (black circles). Each figure corresponds to $10^{5}$ independent systems of size $N=512$. The probability distribution is decomposed into the sum of a Gumbel distribution (dashed line) and a gaussian distribution (gray area). The black continuous line is the sum of the Gumbel and of the gaussian distributions to compare with the distribution (black circles) given by the numerical simulations. Figure 2 (a) corresponds to the time $t=0.4 t_{c}$. The gaussian component is barely seen. Figure 2 (b) corresponds to the time $t=0.8 t_{c}$. As the system approaches the critical time, the weight of the gaussian component increases.

- $t=t_{c}$

At the critical time, the $s_{\max }$-distribution is expected to be much more complicated. However, it is still well approximated with the sum of a Gumbel and of a gaussian distribution (see Figure 3). Remarkably, one finds that the weights associated to each distribution are fairly equal (i.e. the respective areas of the curves are similar). We can then suggest, in the present case, that the critical point is characterized by the equality of the respective weights of the Gumbel and gaussian contributions. This point will be discussed below.

- $t_{c}<t<t^{\star}$

When the system state is in the post-gelation phase, one expects the gaussian contribution to be the most important part of the order-parameter distribution. This is exemplified on the Figure 4, on which the gaussian part (gray area) is clearly dominant. As expected, the Gumbel part disappears progressively when the time moves away from the gel time (Figure 4 (e)). 


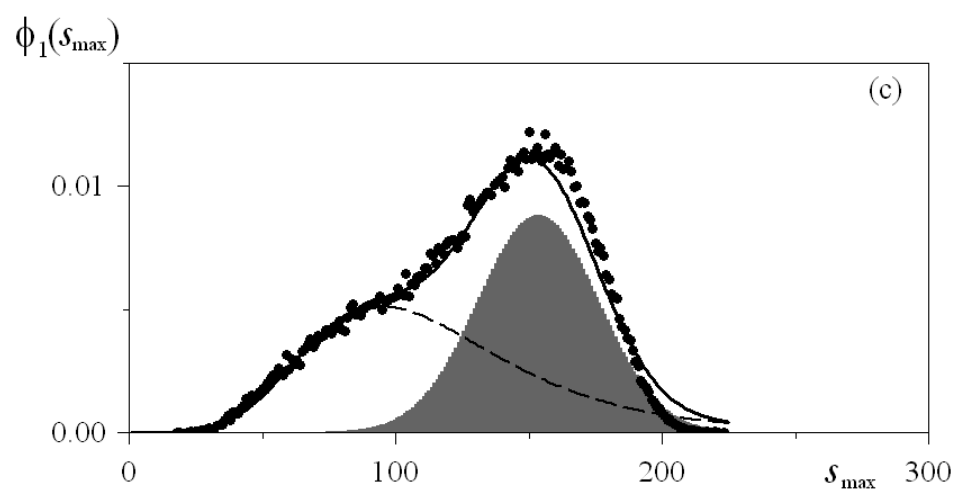

Figure 3: Same analysis as for the Figure 2, for $10^{5}$ independent systems of size $N=512$ at the gel-time of the infinite system, that is: $t=t_{c}$. In this special case, the area of the Gumbel distribution (dashed line) is found to be equal to the area of the gaussian distribution (gray area). Then, both distributions contribute to the $s_{\max }$-distribution with equal weight.

\subsection{Conclusions from the numerical study}

The numerical results suggest a general scenario about the correlations in the system with the extremal order parameter. Namely, if we expand the order-parameter distribution, $\phi_{1}\left(s_{\max }\right)$ as:

$$
\phi_{1}\left(s_{\max }\right) \simeq w_{G u} f_{G u}\left(s_{\max }\right)+w_{G a} f_{G a}\left(s_{\max }\right)
$$

where $f_{G u}$ and $f_{G a}$ are respectively a Gumbel and a gaussian distribution, then, the quantity:

$$
\eta=\frac{w_{G a}-w_{G u}}{w_{G a}+w_{G u}}
$$

is a measure of the distance to the critical point, or, equivalently, $\eta$ is directly related to the value of the correlation size.

According to the general argument given above and the results on the Smoluchowski aggregation model - as exemplified on the Figure 5 -, one can propose the following features for the various values of $\eta$ for a finite system:

- $\eta=1$ when the finite-system state is in the ordered phase

- $\eta=-1$ when the finite-system state is in the disordered phase

- $\eta=0$ coincides with the critical point for the infinite system

- $-1<\eta<1$ characterizes the pseudo-critical domain, where the correlations play a paramount role in the system state

Note that the particular value $\eta=0$ seems to appear for the critical point of the infinite system (in the Figure 5, it occurs for $t / t_{c}=1$ ), and not for the point where the fluctuations of the order parameter are the largest (in the Figure 5 , it should be at $t / t_{c}=1.3$, as shown on Figure 1). 

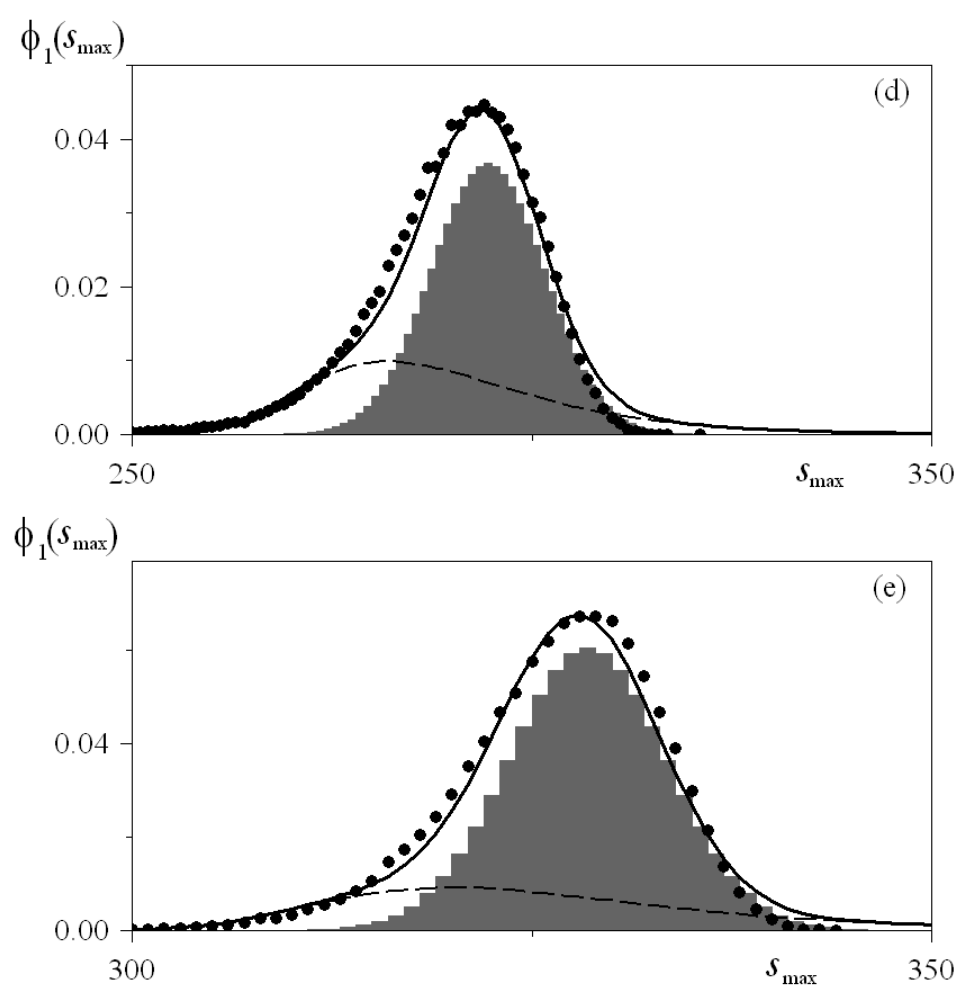

Figure 4: Same analysis as for the Figure 2, for $10^{5}$ independent systems of size $N=512$ in the post-gelation domain $t>t_{c}$. Figure 4 (d) corresponds to the time $t=1.4 t_{c}$. Figure 4 (e) corresponds to the time $t=1.6 t_{c}$. As the time goes, the weight of the Gumbel component (dashed curve) decreases.

\section{4 robustness of the analysis}

Various tests were performed to check how robust is the resent analysis. For example, we added a fragmentation term to the Smoluchowski aggregation equations (4.1). Generally, it results in a saturation of the standard deviation $\sigma$ of the largest fragment when steady-state is reached, that is for time $t \rightarrow \infty$. However, the critical behavior at the finite time is kept when the initial conditions are regular (e.g. monodisperse system of fragments of size 1 ; this corresponds to the initial condition as the extreme disordered state). Then, we observe that the analysis in the sum of a Gumbel and of a gaussian distributions, stays the same, and is still able to detect the critical point of the infinite matter [23]. In the case of aggregation/fragmentation process, detailed balanced can be essential [24], but for the present analysis, no effect was noticeable when choosing a system in which detailed balance was not realized. Also the Smoluchoswki kernel $K_{0} i j$ was changed in $K_{0} i^{2 / 3} j^{2 / 3}$, that corresponds to different coalescence cross-sections, but still exhibits a critical gel time. In this case too, the decomposition in sum of two standard distributions worked well in the pseudo-critical domain around the gel time.

\section{5. how to get information about the real fragmentation process}

In the analysis above, we were interested in detecting a possible critical point of the infinite system from the overall shape of the order-parameter distribution. A more subtle point is to know 


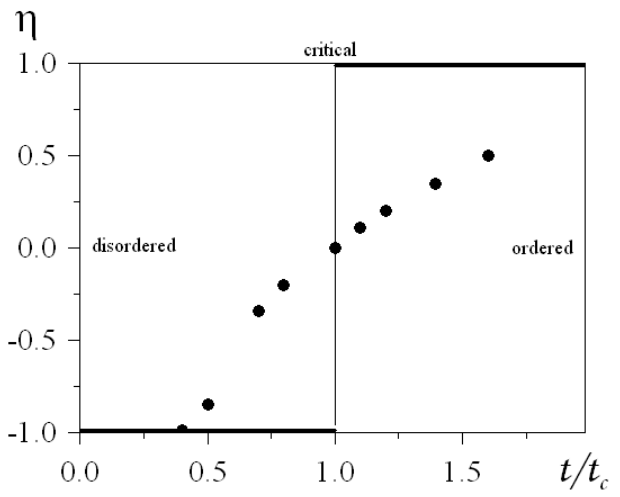

Figure 5: Sketch of the $\eta$ values versus the reduced time $t / t_{c}$ for the Smoluchowski aggregation model (black circles). When $\eta=-1$, the order parameter distribution, $\phi_{1}\left(s_{\max }\right)$, is the Gumbel distribution. When $\eta=1, \phi_{1}$ is the gaussian distribution. In this model, the finite-system state goes gently from Gumbel-like to Gauss-like, passing through the particular value $\eta=0$ at the critical time, $t / t_{c}=1$. The bold line in the shape of a step function passing discontinuously from the value -1 to the value +1 at $t / t_{c}=1$, is the expected behavior of $\eta$ for the infinite system.

something about the mechanisms which leads to the fragments. In principle, one can obtain precise information investigating the second largest fragment, $s_{2}$. Indeed, if the systems created instantaneously all the fragments (a process called 'multifragmentation' [26]), the distribution of the $k^{\text {th }}$ largest fragment, $s_{k}$, in the disordered phase should be [25]:

$$
\begin{aligned}
\phi_{k}\left(s_{k}\right) & =\frac{1}{(k-1) !} \frac{1}{b_{m}} e^{-k z_{k}-e^{-z_{k}}} \\
z_{k} & =\frac{s_{k}-a_{m}}{b_{m}}
\end{aligned}
$$

in which the scaling coefficients $a_{m}$ and $b_{m}$ are the same as for the largest-fragment case, that is (2.5).

Another option for the mechanism is that fragmentation occurs step by step: the system splits into several fragments, then each of the fragments breaks up in several pieces and so on. After $v$ steps of successive fragmentations, the largest fragment in the system will be probably the result of the fragmentation of the largest piece in the step $v-1$. Then the distribution of $s_{\max }$ will be a Gumbel distribution, with the coefficients $a_{m}$ and $b_{m}$ corresponding to the parameters of the parentpiece. Nothing would change between the scenario of the multifragmentation, and the scenario of the sequential fragmentation [26]. However, the distribution of the second largest fragment is different, because this fragment is almost surely the largest fragment of its own parent-piece. Then, one expects the distribution of $s_{2}$ to be also a Gumbel distribution with different values of the parameters $a_{m}, b_{m}$. More precisely, the two options will write respectively:

$$
\begin{aligned}
b_{m} \phi_{1}\left(s_{\max }\right) & =e^{-z_{1}-e^{-z_{1}}} ; b_{m} \phi_{2}\left(s_{2}\right)=e^{-2 z_{2}-e^{-z_{2}} \quad \text { (multifragmentation) }} \\
z_{1} & =\left(s_{\max }-a_{m}\right) / b_{m} ; z_{2}=\left(s_{2}-a_{m}\right) / b_{m} \\
b_{m} \phi_{1}\left(s_{\max }\right) & =e^{-z_{1}-e^{-z_{1}}} ; b_{m} \phi_{2}\left(s_{2}\right)=e^{-z_{2}^{\prime}-e^{-z_{2}^{\prime}} \quad \text { (sequential-fragmentation) }} \\
z_{1} & =\left(s_{\max }-a_{m}\right) / b_{m} ; z_{2}^{\prime}=\left(s_{2}-a_{m}^{\prime}\right) / b_{m}^{\prime}
\end{aligned}
$$


There are two differences. One is in the coefficient 2 in the first exponential function of the $\phi_{2}$ function (note that this coefficient is in principle easy to detect as an extra factor 2 in the slope of $\ln \phi_{2}\left(s_{2}\right)$ versus $s_{2}$ for the large values of $s_{2}$, when plotted in the scaled variable $\left.z_{2}\right)$. The other difference is about the values of the couple of parameters $a_{m}^{\prime}, b_{m}^{\prime}$ (for $s_{2}$ variable) compared with $a_{m}, b_{m}$ (for the $s_{\max }$ variable).

Generally, it is difficult to obtain precise probability distribution of the third or smaller fragments, but if information is available, it can be used the same way to check more carefully the aggregation/fragmentation scenario.

\section{Conclusion}

Finite systems cannot exhibit critical phenomena, though correlations play a relevant role within an extended range of the parameter controlling the system state. This range of values is called the pseudo-critical domain, and can be defined precisely.

In the present work, we studied the case of systems where the only available information is about fluctuations of the extremal order-parameter. Two critical models in the same universality class, are discussed in this context: the percolation - which is at-equilibrium model -, and the Smoluchowski aggregation model - which exhibits a dynamical phase transition - . Both models give a novel coherent insight about the structure of the order-parameter distribution function in the pseudo-critical domain. Because the system is finite, the visible system state appears to be sometimes in the disordered state, and sometimes in the ordered state, when the control parameter is close to the critical point. Consequently, the effective order-parameter distribution is well fitted by a weighted sum of a Gumbel distribution (characteristic of the disordered state) and of a gaussian distribution (characteristic of the ordered state). The parameter $\eta$ - which is essentially the relative ratio between the Gumbel and the gaussian contributions -, is proposed to measure the distance of the average system state to the critical state of the infinite system. This new parameter can easily be calculated from the order-parameter distribution of the finite system, and its proper value gives a definite information about the strength of the correlations acting in the system. Moreover, one of the observable parameters $\left(b_{m}\right)$ gives an estimation of the actual correlation size in the system.

When one can have access at the same time to the largest and the second largest fragment, then, the probability distribution of the second largest fragment gives quantitative information about the mechanisms which produced the fragments.

\section{Acknowledgments}

During my activity on this subject, I have profited from many discussions and collaborations with M. Płoszajczak and J. Frankland from GANIL (France), and I thank them warmly. We are together preparing a paper about the analysis presented above, applied to experimental data of heavy-ions multifragmentation at intermediate energies. 


\section{References}

[1] A.D. Bruce, J. Phys. C 14 3667-3688 (1981); M.M. Tsypin and H.W.J. Blöte, Phys. Rev. E 6273 (2000).

[2] R. Botet and M. Płoszajczak, Universal Fluctuations - the phenomenology of hadronic matter-, World Scientific Lecture Notes in Physics, vol. 65, World Scientific, Singapore, 2002.

[3] D. Nicolaides and A. D. Bruce, J. Phys. A 21, 233 (1988).

[4] H. Fischer, History of the Central Limit Theorem: From Classical to Modern Probability Theory, Springer, New York (2010).

[5] B. Bollobàs and O. Riordan, Percolation, Cambridge University Press, Cambridge (2006).

[6] D. Stauffer and A. Aharony, Introduction to Percolation Theory (2nd ed.), Taylor \& Francis, London (1994).

[7] R. A. Fisher and L. H. C. Tippett, Proc. Cambridge Phil. Soc. 24, 180-190 (1928) ; B. V. Gnedenko, Annals of Mathematics 44, 423-453 (1943).

[8] S. Coles, An Introduction to Statistical Modeling of Extreme Variables, Springer-Verlag, London (2001).

[9] J. Pickands, Ann. Probab. 14, 996 (1986).

[10] I. Gomez, in Extreme Value Theory and Applications, eds. J. Galambos, J. Lechner and E. Simiu, Kluwer Acad. Publ., The Netherlands, 1994.

[11] E. J. Gumbel, Statistics of Extremes, Columbia University Press, New York (1958).

[12] G. A. Martynov, Teoret. Matemat. Fisika 156, 454-464 (2008) (English translation: Theoret. Math. Phys. 156, 1356-1364 (2008)).

[13] one can derive a more precise result: if the cut-off of the fragment-size distribution, $f(s)$ behaves as $\sim \exp \left(-s^{c}\right)$, then the combination $\left(b_{m} a_{m}^{c-1}\right)^{1 / c}$ gives an estimation of the correlation size $\xi$ independently of the multiplicity.

[14] M. Z. Bazant, Phys. Rev. E 62, 1660-1669 (2000).

[15] R. Van der Hofstad and F. Redig, J. Stat. Phys. 122, 671-703 (2006).

[16] D. Wilmsa, A. Winklera, P. Virnaua and K. Binder, Computer Physics Communications 182, 1892-1895 (2011); the present definition for 'pseudo-critical domain' must not to be confused with the 'pseudo-critical temperature' which is the temperature for which the susceptibility reaches maximum, e.g.: S. Wiseman and E. Domany, Phys. Rev. E 52, 3469-3484 (1995).

[17] H. E. Stanley, Introduction to Phase Transitions and Critical Phenomena, Oxford Science Publications, New York (1987).

[18] R. Botet and M. Płoszajczak, Phys. Rev. Lett. 95, 185702-4 (2005).

[19] Ph. Biane, J. Pitman and M. Yor, Bull. Amer. Math. Soc. 38, 435-465 (2001).

[20] R. Botet, J. Phys.: Conf. Ser. 297, 012005-14 (2011).

[21] R. L. Drake, A general mathematical survey of the coagulation equation, in 'Topics in Current Aerosol Research', G. M. Hidy and J. R. Brock, eds., International Reviews in Aerosol, Physics and Chemistry, Pergamon, Oxford, 201-376 (1972). 
[22] M. V. Smoluchowski, Physik. Z. 17, 557-585 (1916).

[23] R. Botet, "Which hidden correlations in the biggest-fragment probability distribution?", oral presentation at 'The Seventh Workshop on Particle Correlations and Femtoscopy', September 20 - 24 2011, University of Tokyo, Japan.

[24] A. N. Gorban and G. S. Yablonsky, Chemical Engineering Science 66, 5388-5399 (2011).

[25] E. J. Gumbel, Annales de l'I.H.P. 5, 115-158 (1935).

[26] B. Borderie and M.-F. Rivet, Progress in Particles and Nuclear Physics 61, 551-601 (2008). 